\title{
MULTIPLE CONGENITAL HEART ABNORMALITIES
}

\author{
S. Lazarov', G. Bogdanov², R. Nikolov² \\ ${ }^{1}$ Department of Pathophysiology, Medical University - Sofia, Bulgaria \\ ${ }^{2}$ Department of Pharmacology and Toxicology, Medical University - Sofia, Bulgaria
}

\begin{abstract}
Congenital heart abnormalities are rare and most often occur separately. These structural and functional disorders can cause a range of diseases that vary in severity, from mild or even asymptomatic to severe and life-threatening. Occasionally these conditions could cause no symptoms in which case they are found accidentally when the patient is examined for other reasons or during autopsy. In this article we present the case of a 47-year-old male patient with triple congenital heart anomaly detected in two consecutive tests performed in pre-hospital medical care.
\end{abstract}

Key words: congenital heart abnormalities, dual AV-node physiology, coronary artery anomaly $(C A A)$, left ventricular diverticulum, electrophysiological study (EPS), invasive examination, ischemic heart disease (IHD), atrioventricular nodal reentrant tachycardia (AVNRT)

Corresponding author: G. Bogdanov, e-mail: dr.georgi.bogdanov@gmail.com

\section{INTRODUCTION}

W e present a 47-year-old male patient with three congenital heart anomalies: dual AV - node physiology (found with an electrophysiological study (EPS) after symptoms of atrioventricular nodal reentrant tachycardia (AVNRT)), coronary artery anomaly (CAA), and diverticulum of the left ventricle (found with selective coronary angiography (SCAG) and left ventriculography performed due to signs of ischemic heart disease with unstable angina).

Dual AV-node physiology. This anomaly is found in around $5 \%$ of the total population. In some conditions this anomaly might induce atrioventricular node reentry tachycardia (AVNRT). The symptoms of AVNRT include: palpitations, anxiety, restlessness, dizziness, chest discomfort or pain, shortness of breath and seizures. Sometimes AVNRT might be asymptomatic $[1,4,6,15,17]$.
Congenital coronary artery anomaly (CAA). These anatomical anomalies are found in less than 1\% of the total population. CAA is often accompanied by other congenital heart malformations. In many cases CAA is asymptomatic and is found during autopsy. The symptoms vary depending on the type and severity of the defect. CAA might be associated with sudden death $[3,11,13]$.

Congenital left ventricular (LV) diverticulum. This is a rare congenital malformation affecting $0.26-0.4 \%$ of the total population. It is found in 3 out of 750 autopsies of people with sudden heart death. It is characterized by a localized outpouching from the cardiac chamber and is built from endocardium, myocardium and epicardium. The congenital left ventricle diverticulum has a normal contractility unlike the ventricular aneurysm. Usually, it is a single defect (although in rare cases there might be multiple congenital left ventricular diverticula) localized at the apical part of 
the chamber, but it might also be with different localization $[2,5,7,8,9,10,12,14,16]$.

The congenital left ventricle diverticulum is a result of the formation of a weak zone in the wall of the left chamber during the 2nd or 3rd week of the embryonic life. Possible reasons for the development of this defect are intrauterine viral infections, muscular and connective tissue defects and extreme stimulation of the cells. Usually, the patients experience no symptoms. Complications like thromboembolism, infectious endocarditis, ventricular arrhythmias, rupture of the ventricles or sudden death might be among the first signs of this anomaly $[2,5,7,8,9$, $10,12,14,16]$.

\section{DESCRIPTION OF THE CASE}

The patient presented to the physician's office with complaints of recurrent headaches, dizziness, fainting, palpitations, precordial chest pain and tingling of the hands, feet and face. The physician established: pulse frequency 160 beats/min, BP 100/60 mm Hg. The ECG showed supraventricular tachycardia and heart rate 160 beats/min (Figure 1). The patient had a history of traumatic pneumothorax of the left lung with drainage 4 years ago. He also had recurrent episodes of syncope beginning 3 years ago (around ten episodes). The patient was not taking any drugs. The patient smoked regularly (30-40 cigarettes daily). The function of the thyroid gland was normal.

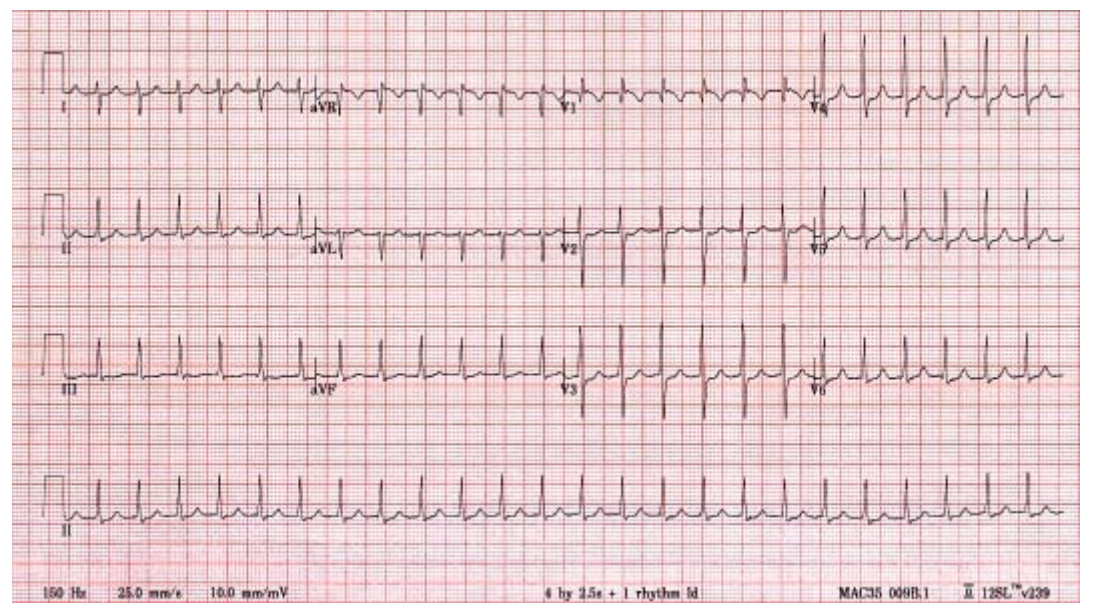

Fig. 1. ECG - The ECG showed supraventricular tachycardia and heart rate 160 beats/min

The patient was then directed to an emergency center where the arrhythmia was incorrectly diagnosed as atrial fibrillation. Successful pharmacological treatment was performed with the use of Amiodarone 300 $\mathrm{mg} / 6 \mathrm{ml}$ i.v. This led to permanently retained sinus rhythm. The patient was consulted by a cardiologist who directed him to a planned electrophysiological study (EPS).

An echocardiography was performed. It showed normal volume and sizes of the cardiac cavities, normal ventricular function and contractility. No pericardial effusion was found. Aortic valve ring $-21 \mathrm{~mm}$, sinus of Valsalva $-31 \mathrm{~mm}$, ascending aorta $-28 \mathrm{~mm}$, left atrium - $36 \mathrm{~mm}$, interventricular septum (IVS)/ left ventricular posterior wall (LVPW) - 10/9 mm, LV end-diastolic dimension/end-systolic dimension $(E D D) /(E S D)-51 / 39 \mathrm{~mm}$, ejection fraction - 64\%, end-diastolic volume/end-systolic volume (EDV/ ESV) - 110/33 ml, ejection fraction - 69\%, mitral regurgitation $0+$, right ventricular wall $-4.5 \mathrm{~mm}$, and at base - $38 \mathrm{~mm}$, pulmonary blood pressure - $133 \mathrm{~mm}$ $\mathrm{Hg}$, left atrium $-36 / 28 / 38 \mathrm{~mm}$, right atrium $-41 / 30$ $\mathrm{mm}$, mitral valve - fibrotically altered, with thickened cusps and single calcifications on the chords and the cusps, E/A 1.65. The rest of the valves were intact.

Electrophysiological study. Dual AV-node physiology was determined (presence of slow conduction $\alpha$-pathway with long refractory period and fast conduction $\beta$-pathway with short refractory period). A classical slow-fast AVNRT was induced. Successful ablation of the slow pathway of the AV-node with 4 $\mathrm{mm}$ irrigated catheter Biosense Cool Flow was performed. No retrograde conduction on the $490 \mathrm{~ms}$ was noted after the procedure was over. Normal anterograde $\mathrm{AV}$-node conduction at the end of the procedure with Wench point 460 ms was registered. Infusion therapy with Heparin 5000 $\mathrm{IU}$ and Lidocaine $200 \mathrm{mg} / 10 \mathrm{ml}$ was initiated. The procedure time was around $60 \mathrm{~min}$, observation time $10 \mathrm{~min}$, DAP $9544 \mu \mathrm{Gy} / \mathrm{m}^{2}$.

Holter-ECG. Because of recurrence of the symptoms a Holter monitor was installed and it detected attacks of atrial tachycardia, short attacks of atrial fibrillation and frequent supraventricular and ventricular extrasystoles.

Electrophysiological study. Because of the before mentioned arrhythmias a second EPS was performed. Normal AV-node conduction was determined. No AVNRT or other long lasting SV tachyarrhythmias were induced. DAP $2521 \mu \mathrm{Gy} / \mathrm{m}^{2}$.

Treatment with Propafenone $3 \times 150$ mg was prescribed. The condition was stabilized for a while, but attacks of palpitations occurred again. The patient decided himself to stop the treatment. 
One year later the patient presented to a cardiology clinic with complaints of darkening before his eyes, pre-cordial pain and tingling of the hands. These symptoms were persistent for the last hour. The patient's heart rate was 70 beats $/ \mathrm{min}$, the ECG showed rSr-forms in V1-V3. Insignificant ST-elevation with concave upward and coronary T-waves in V3-V6 were determined. Hemodynamic stabilization was performed and although there was initial GRACE RS -121 , an early invasive procedure was done.
Invasive study. ARD $6 \mathrm{~F}$ access. Right type of coronary circulation. LM, LAD, LCX and RCA without stenoses or data about thrombosis. Mitral valve with fibrotic changes, thickened cusps and single calcifications on the cusps and the chords, insufficiency $0+$. Aortic valve with fibrotic changes. Tricuspid valve was intact. Presence of coronary anomaly ( $L C X$ begins as a first branch of RCA) (Figure 2) and apical left ventricle diverticulum (Figure 3). Infusion with Isocor $2.5 \mathrm{mg}$, Heparin $5000 \mathrm{IU}$ and NGL $100 \mu \mathrm{g}$ was initiated. DAP $834 \mu \mathrm{Gy} / \mathrm{m}^{2}$.
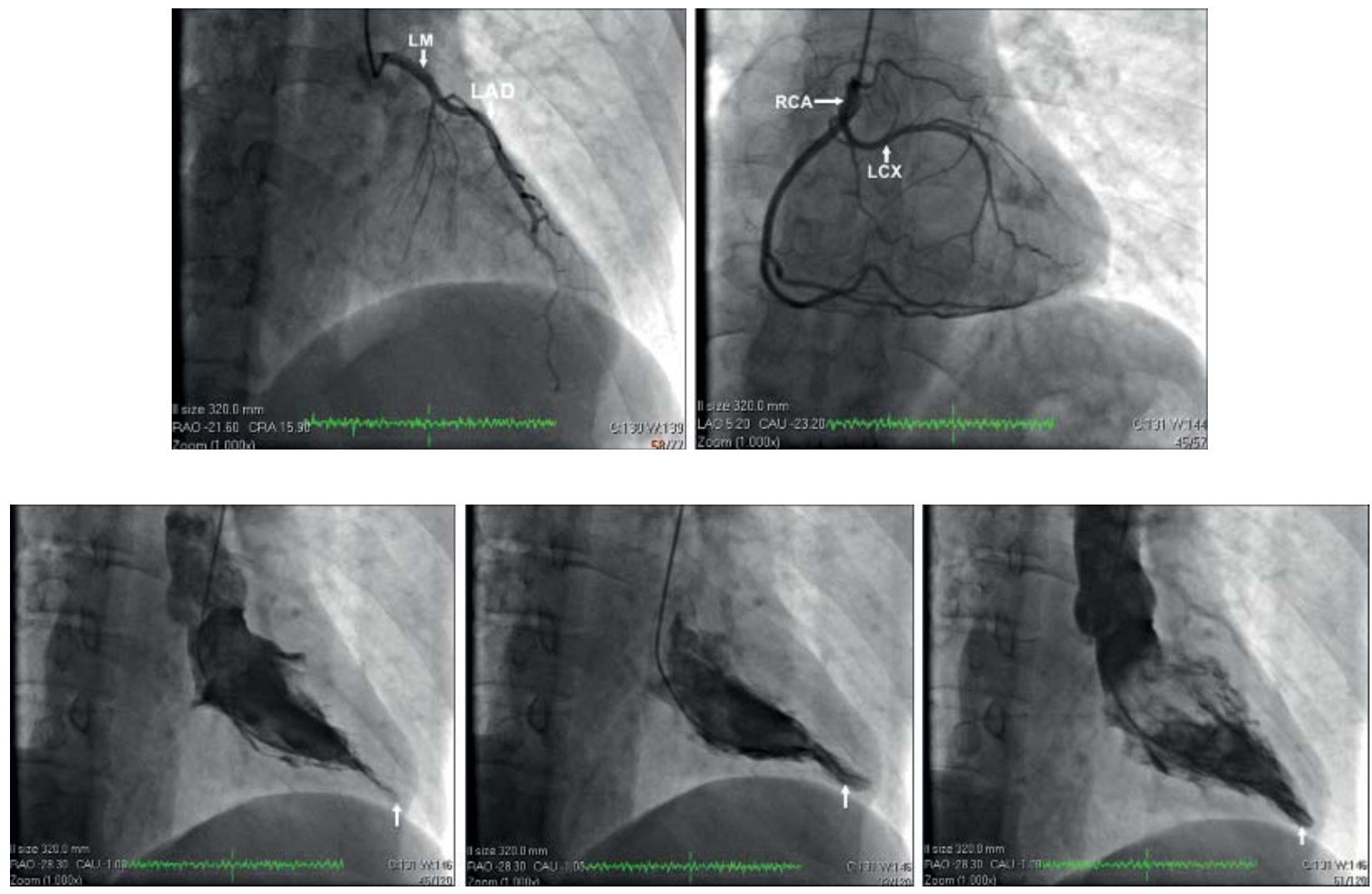

Fig. 2 and 3. Description of the invasive procedure - Presence of coronary anomaly (LCX begins as a first branch of RCA) (fig. 2) and apical left ventricle diverticulum (fig. 3)

\section{DISCUSSION OF THE CASE}

The presence of dual AV-node physiology in some conditions (stress, physical exercise, hyperthyroidism, excessive consumption of coffee, tea, alcohol, impaired metabolism of calcium, potassium and magnesium and others) might trigger AVNRT. It runs with a variable ventricular rate $(140-280$ beats $/ \mathrm{min})$ and different in degree hemodynamic alterations which are responsible for the symptoms of the patient [1, $4,6,15,17]$.

The high degree AVNRT causes arterial hypotension and impairs the hemodynamics. The disrupted brain circulation leads to symptoms of syncope in the patient (dizziness, blackening in front of the eyes, pain and tingling of the arms, legs and the face, short loss of consciousness - seconds to a minute, according to the patient it always comes after palpitations) [ 1 , $4,6,15,17]$.

The ablation of the slow pathway leads to a definitive cure of the AVNRT $[1,4,6,15,17]$. Due to the bad habits of the patient (tobacco smoking), however, there is predisposition to atrial or ventricular extrasystoles and atrial tachyarrhythmias (established with several consecutive ambulatory studies with Holter-ECG). The treatment with Propafenone $3 \times 150 \mathrm{mg}$ turned out to be ineffective. Therefore, in accordance with the heart rate, Sotalol $2 \times 80 \mathrm{mg}$ was prescribed. The therapeutic effect was good: the patient had no symptoms, and several consecutive ambulatory tests with Holter monitor showed no pathology in the rhythm and conduction. 
In case of a high-frequency arrhythmia with/or arterial hypotension the coronary anomaly $[3,11,13]$ and the left ventricle diverticulum $[2,5,7,8,9,10,12,14,16]$ might provoke impairment of the coronary blood flow, by its redistribution (development of "steal phenomenon"). This leads to left ventricle myocardial ischemia (decrease in the quantity of oxygen and nutrients which reach the LV myocardium). This explains the sudden symptoms in the patient (pre-cordial anginalike pain).

\section{Conflict of interest}

The authors declare lack of circumstances that could lead to a conflict of interest, including financial commitment.

\section{REFERENCES}

1. Al-Sayegh A, Gondimalla VD, Shukkur AM. Atrioventricular Nodal Re-entrant Tachycardia Ablation: Unusual Function of Slow Pathway. Heart Views. 2011; 12(1): 32-34.

2. Romagnoli A, Ricci A, Morosetti $D$ et al. Congenital left ventricular diverticulum: Multimodality imaging evaluation and literature review. J Saudi Heart Assoc. 2015; 27(1): 61-67.

3. Pelliccia A. Congenital coronary artery anomalies in young patients. J Am Coll Cardiol. 2001;37(2):598-600.

4. Ching-Tai Tai, Shih-Ann Chen, Chern-En Chiang, et al. Multiple anterograde atrioventricular node pathways in patients with atrioventricular node reentrant tachycardia. J Am Coll Cardiol. 1996;28(3):725-731.

5. Cianciulli T, Colaso P, Saccheri M et al. Left ventricular diverticulum, a rare echocardiographic finding: Case report and review of the literature. Cardiol J. 2009;16(1):76-81.
6. Demosthenes G. Katritsis and Camm AJ. Atrioventricular Nodal Reentrant Tachycardia. Circulation. 2010;122:831840.

7. Shah D, Kumar CP, Shah MS, Baraiya M. Congenital left ventricular diverticulum. Indian J Radiol Imaging. 2010; 20(3): 211-214.

8. Mardini MK. Congenital diverticulum of the left ventricle. Report of two unusual cases. Br Heart J 1984;51(3):321-6.

9. Hamada M, Teruyama Y, Yamashita $\mathrm{H}$, et al. Congenital Left Ventricular Diverticulum. Circulation. 2013;128: 451-452.

10. Tanaka M, Kawahito K, Kaneda H, Saito S. Congenital left ventricular diverticulum in an adult. European Heart Journal. 2006; 6: 1537.

11. Hauser M. Congenital anomalies of the coronary arteries. Heart. 2005; 91(9): 1240-1245.

12. Okereke OUJ, Cooley DA, Frazier $\mathrm{OH}$. Congenital diverticulum of the ventricle. J Thorac Cardiovasc Surg 1986; 91: 208-14.

13. Angelini P. Coronary Artery Anomalies. Circulation. 2007; 115:1296-1305.

14. Pressoir R, Downing JW. Congenital diverticula of the right ventricle of the heart: a case report. J Natl Med Assoc 1980;72 (3):262-4.

15. Shen EM, Fukuyama O, Herre JM et al. Ventricular taquycardia with congenital ventricular diverticulum. Chest 1991; 100: 283-85.

16. Skapinker S. Diverticulum of the left ventricle of the heart; review of the literature and report of a successful removal of the diverticulum. AMA Arch Surg 1951;63(5):629-34.

17. So Yeon Kim, Joon Beom Seo, Kyung-Hyun Do, et al. Coronary Artery Anomalies: Classification and ECG-gated MultiDetector Row CT Findings with Angiographic Correlation. Radiology and RadioGraphics. 2006; 26(2): 317-332.

Received: May, 2019 - Accepted: June, 2019 